\title{
CYP3A5 polymorphisms in renal transplant recipients: influence on tacrolimus treatment
}

This article was published in the following Dove Press journal: Pharmacogenomics and Personalized Medicine

\author{
Lucy Chen' \\ G V Ramesh Prasad ${ }^{2}$ \\ 'Kidney Transplant Program, \\ St Michael's Hospital, Toronto, ON, \\ Canada; ${ }^{2}$ Division of Nephrology, \\ St Michael's Hospital, Toronto, ON, \\ Canada
}

Correspondence: Lucy Chen 6I Queen Street E, Room 7-016, Toronto, ON M5C 2T2, Canada

Tel +l 4379993520

$\mathrm{Fax}+$ I 4168673740

Email ChenLu@smh.ca

\begin{abstract}
Tacrolimus is a commonly used immunosuppressant after kidney transplantation. It has a narrow therapeutic range and demonstrates wide interindividual variability in pharmacokinetics, leading to potential underimmunosuppression or toxicity. Genetic polymorphism in CYP3A5 enzyme expression contributes to differences in tacrolimus bioavailability between individuals. Individuals carrying one or more copies of the wild-type allele *1 express CYP3A5, which increases tacrolimus clearance. CYP3A5 expressers require 1.5 to 2 -fold higher tacrolimus doses compared to usual dosing to achieve therapeutic blood concentrations. Individuals with homozygous *3/*3 genotype are CYP3A5 nonexpressers. CYP3A5 nonexpression is the most frequent phenotype in most ethnic populations, except blacks. Differences between CYP3A5 genotypes in tacrolimus disposition have not translated into differences in clinical outcomes, such as acute rejection and graft survival. Therefore, although genotype-based dosing may improve achievement of therapeutic drug concentrations with empiric dosing, its role in clinical practice is unclear. CYP3A5 genotype may predict differences in absorption of extended-release and immediate-release oral formulations of tacrolimus. Two studies found that CYP3A5 expressers require higher doses of tacrolimus in the extended-release formulation compared to immediate release. CYP3A5 genotype plays a role in determining the impact of interacting drugs, such as fluconazole, on tacrolimus pharmacokinetics. Evidence conflicts regarding the impact of CYP3A5 genotype on risk of nephrotoxicity associated with tacrolimus. Further study is required.
\end{abstract}

Keywords: calcineurin inhibitor, graft, pharmacogenomics, kidney, genotype

\section{Introduction \\ Tacrolimus}

Tacrolimus (Astellas Pharmaceuticals, Northbrook, IL, USA) is an immunosuppressant of the calcineurin inhibitor class and is considered a cornerstone of maintenance drug therapy after kidney transplantation. Tacrolimus binds to FK506-binding protein 12, which in turn inhibits the action of calcineurin, which is involved in T-cell activation. ${ }^{1}$ Tacrolimus was first introduced for clinical use in transplantation in 1989. Initial studies of tacrolimus demonstrated that it reduced 1-year acute rejection rates and improved 6-months graft survival compared to its predecessor, ciclosporin (Novartis Pharmaceuticals, Basel, Switzerland). ${ }^{2}$ Over the last 3 decades, use of tacrolimus as the calcineurin inhibitor of choice has grown by large margins. As of 2015, in the United States, tacrolimus is used as part of the immunosuppressant regimen for more than $90 \%$ of all kidney transplant recipients. ${ }^{3}$ Tacrolimus is available for oral administration in several formulations. The immediate-release capsule is given twice daily, while the 
extended-release capsule or tablet is administered once daily. Oral granules for suspension are available in Europe. It is also available in intravenous form.

Tacrolimus has a narrow therapeutic index. Tacrolimus toxicity occurs at concentrations slightly above or even within its therapeutic range. Toxic effects include nephrotoxicity, infection, hypertension, hyperkalemia, hypomagnesemia, hyperglycemia, diabetes, tremor, and other neurotoxic effects. On the other hand, underdosing can lead to underimmunosuppression and graft rejection. Therefore, therapeutic drug monitoring of tacrolimus is used regularly in clinical practice with the goal of optimizing the fine balance between graft rejection and drug toxicity. Trough blood concentration ( $\mathrm{C} 0$ ) is the commonly accepted parameter for therapeutic drug monitoring since it predicts risk of both acute rejection and toxicity. $\mathrm{C} 0$ correlates with overall drug exposure as measured by 12-h area under the curve (AUC0-12h). ${ }^{4}$ There is high interindividual variability in tacrolimus pharmacokinetics. Empiric dosing does not result in predictable systemic drug concentrations.

Tacrolimus is absorbed from the distal segments of gastrointestinal tract with highly variable bioavailability ranging from $5 \%$ to $93 \% .^{5}$ Absorption is rapid, and full absorption occurs in 30-60 minutes after administration of the immediate-release formulation, while the extendedrelease formulation requires 120 minutes. ${ }^{6,7}$ Multiple factors modify its bioavailability such as high dietary fat intake and diabetes, both of which reduce drug absorption. Intestinal enzymes also affect the extent of absorption. Intestinal CYP3A enzymes in the upper small intestine contribute to a first-pass effect in which the drug is metabolized within the gut before it reaches the systemic circulation. Additionally, p-glycoprotein within the gut wall actively pumps drug from the intracellular space of intestinal mucosa cells back into the intestinal lumen. Tacrolimus has a small apparent volume of distribution $(\mathrm{Vd}=1-1.5 \mathrm{~L} / \mathrm{kg})$ because it binds extensively (99\%) to erythrocytes and blood plasma proteins. Tacrolimus is mainly metabolized in the liver by CYP3A isoenzymes. The key metabolites are demethylhydroxy-tacrolimus and demethyl-tacrolimus, although up to 13 other metabolites have been described. Tacrolimus excretion is primarily via the biliary route, with $97 \%$ of radiolabelled tacrolimus recovered in feces. Approximately $3 \%$ of tacrolimus is recovered in urine. ${ }^{8}$ Tacrolimus half-life is approximately 15.6 hours in adult kidney transplant recipients. ${ }^{6,7}$

\section{CYP3A subfamily}

Tacrolimus pharmacokinetics is heavily influenced by drugmetabolizing enzymes in the CYP3A subfamily. CYP3A is one of many cytochromes P450, a superfamily of proteins playing a significant role in metabolizing thousands of exogenous and endogenous substances. Individual enzymes within the cytochrome P450 superfamily are categorized by similarities in amino acid sequence. Each enzyme is named according to a family number, a subfamily letter, an individual enzyme number, and an asterisk followed by number for each allelic variant. ${ }^{9}$ The cytochromes (CYP) P450 are found in most tissues in the human body and are involved in hormone synthesis and breakdown, vitamin D synthesis, cholesterol synthesis, and metabolism of potential toxic compounds, including drugs. The CYP3A subfamily is especially important in this latter function as an estimated half of all marketed drugs undergo metabolism via these enzymes. ${ }^{10}$ In humans, four different CYP3A isoenzymes have been identified: CYP3A4, CYP3A5, CYP3A7, and CYP3A43. ${ }^{11}$ In adults, the dominant isoenzyme is CYP3A4, which is expressed in both the gut and the liver. CYP3A5 is likewise present in these tissues, but in addition, it is also present in the prostate and the kidney. CYP3A7 predominates in neonates, but becomes downregulated soon after birth. Its role as a drug-metabolizing isoenzyme is considered negligible in adults. CYP3A43 is less well studied, and so little is known about its functions.

In the case of tacrolimus, the presence of CYP3A4 and CYP3A5 in the intestinal mucosa and in hepatic cells contributes to a first-pass effect as drug molecules are metabolized prior to reaching the systemic circulation. Genetic polymorphism in these 2 enzymes accounts for a significant part of the interindividual variability observed with tacrolimus bioavailability. The best studied genetic variation is in the CYP3A5 gene. $^{12}$

\section{CYP3A5 genotypes and their frequencies}

Up to 9 different alleles have been identified in the CYP $3 A 5$ gene (Table 1). CYP $3 A 5 * 1$ and $* 3$ occur frequently and are the most well studied. Genetic assays generally do not test for the other variants that occur more rarely. The distribution of alleles within the population differs depending on ethnicity (Table 2).

The best studied single-nucleotide polymorphism (SNP) is at position $6986(6986 \mathrm{~A}>\mathrm{G})$, which is located within intron 3 of the CYP $3 A 5$ gene. Individuals carrying at least 1 copy of the A nucleotide are defined as having the $* 1$ allele and are known as CYP3A5 expressers. This allele is associated with higher CYP3A5 enzyme expression. Those with homozygous GG nucleotides at position 6986 are known as *3/*3 carriers and are considered CYP3A5 nonexpressers. ${ }^{21}$ The substitution of $\mathrm{G}$ for A generates incorrect mRNA splicing, leading to an 
early stop codon that results in a nonfunctional protein. In the seminal study by Kuehl et al, ${ }^{22}$ all Caucasian and most African American patients with low concentrations of intestinal or hepatic CYP3A5 ( $<21 \mathrm{pmol} / \mathrm{mg}$ protein) were homozygous for CYP3A5*3. Those with higher CYP3A5 content (between 21 and $204 \mathrm{pmol} / \mathrm{mg}$ protein) possessed at least 1 copy of $C Y P 3 A 5$ $* 1$. Carriers of the * 1 allele have higher overall CYP3A content by approximately three-fold ( $p=0.001$ for Caucasians, $p=0.01$ for African Americans). In these patients, CYP3A5 represents at least $50 \%$ of the total hepatic CYP3A content.

SNPs usually do not occur independently in a given individual. Rather, one observes linkage disequilibrium, in which two or more SNPs interact because genetic material is passed on to the next generation in blocks of DNA known as haplotypes. Studies show that the $C Y P 3 A 5 * 1$ wild-type allele is linked to the $C Y P 3 A 4 * 1 B$ allele. In one study, $67 \%$ of Caucasians and $100 \%$ of African Americans possessing CYP3A4 $* 1 B$ also possessed CYP3A5*1. ${ }^{24}$ CYP $3 A 4 * 1 B$

Table I CYP3A5 alleles

\begin{tabular}{lll}
\hline Allele & Nucleotide variation & Effect on CYP3A5 protein $^{\mathbf{a}}$ \\
\hline$* 1$ & Wild type & Normal function \\
$* 2$ & $27289 \mathrm{G}>\mathrm{T}$ & Limited/no data \\
$* 3$ & $6986 \mathrm{~T}>\mathrm{C}$ & Loss of function \\
$* 4$ & 14665T $>\mathrm{C}$ & Limited/no data \\
$* 5$ & 12952A $>\mathrm{G}$ & Limited/no data \\
$* 6$ & 14690C $>\mathrm{T}$ & Loss of function \\
$* 7$ & 27I3I_27|32insA & Loss of function \\
$* 8$ & 3699G $>\mathrm{A}$ & Limited/no data \\
$* 9$ & 19386C $>\mathrm{T}$ & Limited no data \\
\hline
\end{tabular}

Notes: "Note that "normal" function is not indicative of the most common phenotype within the general population. In most ethnic groups, absence of functional CYP3A5 is most frequent. Adapted from Birdwell et al. ${ }^{13}$ For any updates to this table or CPIC guideline see: https://cpicpgx.org/guidelines/guideline-fortacrolimus-and-cyp $3 \mathrm{a} 51$

Table 2 Frequency of CYPA5 alleles in different ethnic populations

\begin{tabular}{llll}
\hline \multicolumn{3}{l}{ Frequency of CYP3A5 allele } & \\
\hline Ethnic population & CYP3A5 *I/*I (\%) & CYP3A5 *I/*3 (\%) & CYP3A5 *3/*3 (\%) \\
\hline Caucasian & 1 & $13-17$ & $82-86$ \\
Black & $37-45$ & $40-54$ & $9-15$ \\
Indian & $2.5-11$ & $38-57$ & $32-60$ \\
Chinese & 7.7 & 44.8 & 47.4 \\
\hline
\end{tabular}

Notes: Data from Barry and Levine, ${ }^{1}$ Rojas et al, ${ }^{14}$ Tang et al, ${ }^{15}$ Chen et al, ${ }^{16}$ Boughton et al, ${ }^{17}$ Niioka et al, ${ }^{18}$ Chandel et al, ${ }^{19}$ and Satoh et al. ${ }^{20}$

Table 3 Key terminology and definitions

\begin{tabular}{|c|c|c|c|c|c|}
\hline Patient profile & Allele & SNP & Effect & $\begin{array}{l}\text { Tacrolimus } \\
\text { trough level }\end{array}$ & $\begin{array}{l}\text { Tacrolimus dose } \\
\text { requirement }\end{array}$ \\
\hline \multirow[t]{2}{*}{ CYP3A5 expressers } & $*|/ *|$ & rs6986 AA & mRNA splices correctly, leading to greater & $\downarrow$ & $\uparrow$ \\
\hline & $* 1 / * 3$ & rs6986 AG & quantity of CYP3A5 enzyme & $\downarrow$ & $\uparrow$ \\
\hline $\begin{array}{l}\text { CYP3A5 } \\
\text { nonexpressers }\end{array}$ & $* 3 / * 3$ & rs6986 GG & $\begin{array}{l}\text { Incorrect mRNA splicing leading to } \\
\text { nonfunctional CYP3A5 protein }\end{array}$ & $\uparrow$ & $\downarrow$ \\
\hline
\end{tabular}

Abbreviation: SNP, single-nucleotide polymorphism. 
Another meta-analysis was completed in 2015 by Rojas et $\mathrm{al}^{14}$ that included 37 observational studies looking at outcomes of tacrolimus dose-adjusted trough concentration, acute rejection, acute nephrotoxicity, and chronic nephrotoxicity among kidney transplant patients. Of the 37 studies, 31 reported tacrolimus trough concentration-to-dose ratio. Rojas' study ${ }^{14}$ showed similar findings as the previous meta-analysis. Tacrolimus dose-adjusted trough concentrations were significantly lower in CYP3A5 expressers with a standardized mean difference of 42.46 (95\% CI: $31.12-53.8) \mathrm{ng} / \mathrm{mL}$ per $\mathrm{mg} / \mathrm{kg} / \mathrm{d}$ at 1 week, 56.49 (95\% CI: 40.94-72.03) ng/mL per $\mathrm{mg} / \mathrm{kg} / \mathrm{d}$ at 2 weeks, 50.73 (95\% CI: 38.01-63.45) at 1 month, 70.45 (95\% CI: $56.34-84.56) \mathrm{ng} / \mathrm{mL}$ per $\mathrm{mg} / \mathrm{kg} / \mathrm{d}$ at 3 months, 63.66 (95\% CI: 49.16-78.16) ng/mL per $\mathrm{mg} / \mathrm{kg} / \mathrm{d}$ at 6 months, and 61.26 (95\% CI: 45.99-76.53) ng/mL per mg/kg/d at 12 months.

Age does not appear to be a significant modifier of CYP3A5 genotypic differences in tacrolimus clearance. In one pediatric study of 48 patients, prepubertal children (age $<12$ years) showed significantly higher weight-adjusted tacrolimus dose requirement by approximately two-fold compared to those older than 12 years. This was observed among both the CYP3A5 nonexpresser and the CYP3A5 expresser groups. Additionally, patients with $C Y P 3 A 5 * 1 / * 3$ genotype demonstrated higher tacrolimus dose requirement compared with $C Y P 3 A 5 * 3 / * 3$ genotype, independent of age. ${ }^{29}$

In terms of intraindividual variability in tacrolimus concentrations, the evidence is conflicting. Yong et $\mathrm{al}^{30}$ found that in a small group of healthy volunteers $(n=29)$, nonexpressers had greater variability. The authors attributed this to the fact that nonexpressers are more reliant on CYP3A4 metabolism, which is more prone to enzyme inhibition or induction effects. However, another study compared the degree of variability in tacrolimus concentrations in 118 renal transplant recipients (37 expressers, 81 nonexpressers) and found that both groups had approximately 14\% intraindividual variability, with no difference between the two groups. ${ }^{31}$

The effect of CYP3A5 polymorphism on clearance appears to be drug specific. The other calcineurin inhibitor commonly used in renal transplantation, ciclosporin, undergoes similar metabolic pathways as tacrolimus. Surprisingly, studies show conflicting results with respect to influence of CYP3A5 polymorphism on ciclosporin metabolism. ${ }^{23}$ In a large study of 147 Chinese renal transplant recipients, carriers of the $C Y P 3 A 5 * 1$ allele in fact had lower dose-normalized trough ciclosporin concentrations compared to CYP3A5 $* 3 / * 3$ individuals. ${ }^{32}$ Two other studies had findings in the opposite direction, with those having CYP3A5*1 allele demonstrating higher trough concentration-to-dose ratio. ${ }^{33,34}$
Four additional studies found no difference between CYP3A5 expressers versus nonexpressers. ${ }^{35-38}$ The differing effect of the polymorphism on the two drugs may be due to the lesser contribution of CYP3A5 to ciclosporin metabolism compared to tacrolimus. ${ }^{39}$

There is limited evidence regarding the effect of other CYP3A5 genetic variants on tacrolimus metabolism. In a genome-wide study 197 African American kidney or kidneypancreas transplant recipients, $C Y P 3 A 5 * 6$ and $C Y P 3 A 5 * 7$ were associated with reduced tacrolimus clearance. ${ }^{26}$ These two alleles lead to loss of protein function, similar to $* 3$, and is presumed to affect tacrolimus clearance to a similar degree. In this study, CYP $3 A 5$ polymorphism explained $34.1 \%$ of the variance in tacrolimus dose. Niioka et al ${ }^{18}$ looked at the predictive value of $C Y P 3 A 5$ genotype on tacrolimus dose requirement in the first month posttransplant. Of 9 clinical or pharmacogenetic factors examined, only CYP3A5 genotype, weight, hematocrit, and total clearance of tacrolimus during continuous intravenous infusion were correlated with tacrolimus dose. The contribution of $C Y P 3 A 5$ genotype to tacrolimus dosing increased over the course of the first month posttransplant, accounting for $7.2 \%$ at day 14 and $20.4 \%$ by day 28. In another study, Wang et $\mathrm{al}^{40}$ screened 768 SNPs in genes affecting the metabolism, transport, and calcineurin inhibition pathways of tacrolimus in a group of 96 renal transplant patients. In addition to $6986 \mathrm{~A}>\mathrm{G}$, two additional CYP3A5 SNPs (rs4646457, rs 15524) were identified to be significantly correlated with tacrolimus dose requirement.

\section{Genotype-based dosing}

The effect of $C Y P 3 A 5 * 1$ and $* 3$ alleles on tacrolimus pharmacokinetics is consistently and extensively documented across a multitude of studies over the past 15 years. ${ }^{14,28}$ These findings have led to strong interest in using CYP $3 A 5$ genotype to guide empiric tacrolimus dosing. The goal of genotype-based dosing is to provide empiric dosing that allows rapid achievement of therapeutic drug concentrations, particularly in the initial days after transplant. In one retrospective study, an empiric weight-based starting dose of $0.1 \mathrm{mg} / \mathrm{kg}$ was used to target a therapeutic range of 4-8 $\mathrm{mcg} / \mathrm{mL} .{ }^{16}$ Among CYP3A5 nonexpressers $(C Y P 3 A 5 * 3 / * 3)$, $50 \%$ of patients achieved $\mathrm{C} 0$ within the target range by day 3 of therapy. Among CYP3A5 expressers (CYP $3 A 5 * 1 / * 3$ or $* 1 / * 1$ ), however, only $35.3 \%$ of patients achieved trough concentrations within the therapeutic range by day 3 . Use of therapeutic drug monitoring did allow for rapid dosing correction. By day $7,64.2 \%$ of expressers, compared to $55.4 \%$ of nonexpressers, achieved therapeutic trough concentrations. 
These results suggest that $C Y P 3 A 5$ genotyping is likely more useful if available before kidney transplant.

A prospective randomized controlled trial of CYP $3 A 5$ genotype-based dosing was performed by Thervet et al. ${ }^{41}$ Investigators enrolled 280 de novo renal transplant recipients from 12 sites. Half of the study population was randomized to standard dosing $0.2 \mathrm{mg} / \mathrm{kg} / \mathrm{d}$ and the other half was randomized to genotype-based dosing $0.3 \mathrm{mg} / \mathrm{kg} / \mathrm{d}$ for CYP3A5 expressers and $0.15 \mathrm{mg} / \mathrm{kg} / \mathrm{d}$ for nonexpressers. The proportion of patients with $C Y P 3 A 5 * 3 / * 3$ was high at $78.8 \%$, with only $16.9 \%$ of patients being heterozygous for CYP $3 A 5 * 1 / 3$ and $4.2 \%$ of patients being homozygous with CYP $3 A 5 * 1 / * 1$. These proportions were expected given the predominantly Caucasian population (89.9\%). There was no difference between the standard and genotype-based dosing groups in the frequency of alleles. At day 3 after drug initiation, significantly more patients in the genotype-based dosing group achieved tacrolimus drug concentrations within the therapeutic range (43.2\%) compared with the standard dosing group $(29.1 \%, p=0.03)$. The genotype-based dosing group also required fewer dose modifications, and the median days to achieve target therapeutic range was fewer. There were no differences observed in clinical outcomes including patient and graft survival, incidence of delayed graft function, acute rejection, biopsy-proven acute rejection, new-onset diabetes, or adverse events in the 3 months follow-up period. However, the study was underpowered to detect differences in these outcomes. Findings from Thervet et $\mathrm{al}^{42}$ were not replicated in a more recent randomized controlled trial of similar design. In this second study, 240 patients living donor kidney recipients received either genotype-based dosing or weight-based dosing of tacrolimus immediately after transplant. The investigators found no difference between the two groups in proportion of patients within target therapeutic range at day 3 after transplant (37.4\% weight-based dosing versus $35.6 \%$ genotype-based dosing, $p=0.79$ ). The authors propose the discrepancy in findings compared to Thervet's study ${ }^{42}$ may be related to differences in patient populations. In the Thervet study, ${ }^{42}$ deceased donor kidney recipients started tacrolimus up to 7 days posttransplant, while in the other study all patients initiated tacrolimus immediately posttransplant. Interindividual differences due to gastrointestinal motility or corticosteroid dose in the first week after transplant may overshadow the effect of CYP $3 A 5$ genotype.

The Clinical Pharmacogenetics Implementation Consortium published guidelines in 2015 with recommendations for CYP3A5 genotype-based dosing of tacrolimus. ${ }^{13,43} \mathrm{It}$ should be noted that these guidelines do not recommend for or against performing genotyping for tacrolimus dosing, given a lack of clear clinical benefit. Rather, the Clinical Pharmacogenetics Implementation Consortium is an international group with interest in facilitating clinical utility of pharmacogenetic testing if results are available.

\section{Which genotype is relevant: recipient or donor?}

As it is CYP3A5 intestinal and hepatic content that mediates differences in tacrolimus bioavailability, it is the kidney transplant recipient - rather than donor-genotype that exerts the influence on drug pharmacokinetics. ${ }^{44}$ On the other hand, donor genotype dictates CYP3A5 expression within the kidney. In the kidney, CYP3A5 is the predominant CYP3A isoenzyme present. In one study of 21 kidney donors, the CYP $3 A 5^{*} 1 / * 3$ genotype was associated with 8 -fold higher microsomal CYP3A5 content in renal tissue, as well as 18 -fold higher CYP3A catalytic activity ( $p=0.0001$ and 0.0137 , respectively). ${ }^{45}$ One may hypothesize that CYP $3 A 5$ genetic polymorphisms affect intrarenal metabolism of tacrolimus and potentially mediate renal effects of the drug.

Calcineurin inhibitors mediate nephrotoxicity via a vasoconstrictive mechanism at the afferent glomerular arteriole, resulting in decreased renal blood flow and decreased glomerular filtration rate. ${ }^{46}$ In one case report, a kidney transplant recipient presented with severe tacrolimus-induced nephrotoxicity on renal biopsy despite low serum concentrations of tacrolimus. ${ }^{47}$ The authors hypothesized that this phenomenon is explained by mismatch between the recipient and donor CYP $3 A 5$ genotype. In this case, the recipient genotype was CYP $3 A 5 * 1 / * 3$, indicating "fast" metabolism mediated by expression of CYP3A5 enzyme. This was observed, whereby the recipient had tacrolimus concentrations below the target range despite doubling of the tacrolimus dose over the course of 1 month. On postoperative day 31, renal biopsy performed for persistent delayed graft function showed prominent isometric vacuolization suggestive of calcineurin inhibitor toxicity. The donor genotype was $C Y P 3 A 5 * 3 / * 3$, representing low enzyme expression locally in the kidney graft. Potentially, this may contribute to higher tacrolimus concentrations in the kidney despite low serum concentrations, leading to nephrotoxicity.

Other authors have tested this hypothesis in kidney and other solid organ transplant recipients, with conflicting results. The study of calcineurin inhibitor nephrotoxicity is challenged by lack of a clear clinical phenotype to define the endpoint. In the largest of these studies, Kuypers et al ${ }^{48}$ 
examined the association between calcineurin inhibitor toxicity and tacrolimus $\mathrm{C} 0, \mathrm{AUC} 0-12 \mathrm{~h}$, dose, and CYP3A5 genotype. Calcineurin inhibitor toxicity was defined as de novo arteriolar hyalinization on biopsy histology. Three hundred four participants were included. As expected, the CYP3A5 expressers showed a trend for higher tacrolimus dose requirement compared to nonexpressers. Higher tacrolimus dose requirement predicted occurrence of calcineurin inhibitor (CNI) toxicity, with $25 \%$ of patients on $>0.2 \mathrm{mg} / \mathrm{kg} / \mathrm{d}$ exhibiting toxicity, versus $16.2 \%$ of patients on $0.1-0.2 \mathrm{mg} / \mathrm{kg} / \mathrm{d}$ and only $4.5 \%$ of those requiring $<0.1 \mathrm{mg} / \mathrm{kg} / \mathrm{d}(p<0.0001)$. Systemic tacrolimus exposure, as measured by $\mathrm{C} 0$ and $\mathrm{AUC}$, was not predictive of $\mathrm{CNI}$ toxicity risk. Rather, presence of a $C Y P 3 A 5^{*} 1$ allele was associated with higher risk of CNI toxicity (32.4\% versus $15.2 \%, p=0.01)$ compared to those homozygous for $C Y P 3 A 5^{*} 3 / * 3$. The authors raise the possibility that the higher intrarenal metabolism of tacrolimus in $C Y P 3 A 5^{*} 1$ individuals increases tacrolimus metabolite content in the kidney. However, it is unclear if and how tacrolimus metabolites cause CNI toxicity.

In two other studies, one with cardiac recipients and one with renal recipients, there was no correlation between CYP3A5 genotype and nephrotoxicity. ${ }^{49,50}$ Two other small studies of liver and kidney allograft recipients found the opposite and that the $C Y P 3 A 5 * 1$ allele was protective against CNI nephrotoxicity. ${ }^{51,52}$ A meta-analysis of 5 studies $(\mathrm{n}=867)$ evaluating effect of CYP3A5 genotype on chronic nephrotoxicity was inconclusive. ${ }^{14}$ It neither demonstrated nor excluded an effect of genetic polymorphism on the risk chronic nephrotoxicity (odds ratio [OR]: 1.81 , 95\% CI: 0.89-3.68). The same authors also pooled results from 3 studies $(n=363)$ assessing risk of acute nephrotoxicity and determined that $C Y P 3 A 5$ polymorphism had no impact (OR: $0.9,95 \%$ CI: $0.45-1.83) .{ }^{14}$

In a small study of 25 African American healthy volunteers, $C Y P 3 A 5$ genotype was correlated with blood pressure, with the $C Y P 3 A 5^{*} 1 / * 1$ genotype having a mean blood pressure $19.3 \mathrm{mmHg}$ higher than those with CYP3A5 $* 3 / * 3$ genotype. ${ }^{45}$ In a study of renal transplant recipients, the $C Y P 3 A 5 * 1$ allele carriers likewise had higher adjusted systolic and diastolic blood pressure at 6 months and 24 months posttransplant compared to noncarriers, although the difference was not statistically significant. ${ }^{53}$ There was also a trend for a higher number of antihypertensives used among the $C Y P 3 A 5 * 1$ allele carriers, although this was not statistically significant either.

\section{Differences in tacrolimus clearance due to drug formulation}

Tacrolimus is available in two oral formulations, either immediate-release tacrolimus (IR-Tac), administered twice daily, or extended-release tacrolimus (ER-Tac), administered once daily. The drug manufacturer (Astellas, Tokyo, Japan) recommends that stable patients can be converted between the two different formulations using 1:1 ratio. ${ }^{7}$ The two formulations demonstrated similar rates of acute rejection, patient survival, and graft survival up to 12 months posttransplant. ${ }^{54}$ Some evidence suggest that there may be differences between the formulations with regard to the effect of CYP $3 A 5$ genotype on drug clearance.

In one study, Fan et $\mathrm{al}^{55}$ found that in a cohort of 106 newly transplanted patients (ER-Tac $n=45$, IR-Tac $n=61$ ), those taking the ER-Tac formulation required a higher total daily dose to achieve target trough concentrations in the first 21 days compared to those on IR-Tac. ${ }^{55}$ This effect was particularly pronounced in the 40 patients who had a CYP $3 A 5 * 3 / * 3$ genotype. The mean daily dose of ER-Tac was greater than mean daily dose of IR-Tac in the cohort of CYP3A5 nonexpressers at day 14, 21, and 28. In the highexpresser group, the difference in dose requirement was less pronounced. Another study demonstrated the opposite finding among 56 renal transplant recipients who took either ER-Tac $(n=24)$ or IR-Tac $(n=32)$ and underwent 24 -h pharmacokinetic monitoring pretransplant, 1 month, and 1 year posttransplant. ${ }^{56}$ In CYP3A5 expressers, the AUC0-24h was $25 \%$ lower if ER-Tac was used compared to IR-Tac.

In another study at a Canadian transplant center, investigators examined the relationship between CYP $3 A 5$ genotype and dose requirement in 60 stable renal transplant recipients (mean time posttransplant 7.4 years) who converted from IR-Tac to ER-Tac. ${ }^{57}$ Patients were converted using 1:1 ratio initially, with therapeutic drug monitoring performed after conversion to determine subsequent dose adjustment. There were $43(71.6 \%)$ patients who were nonexpressers $\left(C Y P 3 A 5^{*} 3 / * 3\right)$ and 17 patients who were expressers $($ CYP $3 A 5 * 1 / * 3$ and $* 1 / * 1)$. A greater proportion of those who were CYP3A5 expressers required a dose increase after switching to ER-Tac ( $69 \%$ versus $47 \%, p=0.004)$. In addition, the expressers also required a greater degree of dose increase than the CYP3A5 nonexpressers $(45.3 \%$ versus $26.6 \%$ increase in dose, $p=0.003)$. One proposed mechanism is that the extended-release formulation increases gut transit time, thereby exposing the drug molecule to CYP3A5 enzymes in the proximal small intestines for a longer duration. This 
allows interindividual enzyme expression differences to exert greater influence on bioavailability.

\section{Role of CYP3A5 polymorphism in tacrolimus drug interactions}

It is well known that tacrolimus disposition is affected by numerous drug interactions, especially if concomitant medications can induce or inhibit either CYP3A enzyme activity or p-glycoprotein activity. However, not all patients are affected to the same degree by such interactions. Wide interindividual variation is observed both clinically and in pharmacokinetic studies. ${ }^{58,59}$

In one study, genetic polymorphism in CYP $3 A 5$ partially explained interindividual variability in response to a tacrolimus-fluconazole drug interaction. ${ }^{60}$ Fluconazole is an antifungal drug with known inhibitory activity on CYP3A enzymes, and therefore effects increased tacrolimus concentrations in renal transplant patients. In this retrospective study, 29 transplant recipients who had tacrolimus concentrations monitored while taking fluconazole $50-400 \mathrm{mg} / \mathrm{d}$ for median duration of 17 days received genotyping. Among the CYP $3 A 5^{*} 1 / * 3$ carriers, dose-corrected tacrolimus trough concentrations did not change significantly from baseline (1.26 \pm 1.23 fold). The CYP $3 A 5 * 3 / * 3$ homozygotes, however, saw a $3.28 \pm 2.34$ fold increase in dose-corrected tacrolimus trough concentrations, which was statistically significantly different from the heterozygous patients $(p=0.04)$. As a result, the CYP3A5 nonexpressers were at greater risk of supratherapeutic tacrolimus concentrations with $73.9 \%$ of patients having tacrolimus concentration $>15 \mathrm{ng} / \mathrm{mL}$, while only $16.7 \%$ of CYP3A5 expressers experienced the same. In vitro studies have found that the fluconazole inhibits CYP3A4 activity to a far greater degree than CYP3A5. ${ }^{6}$ The results of this study may be explained by the fact that CYP3A5 expressers can rely on an "escape pathway" in the form of CYP3A5 when fluconazole exerts inhibitory action on CYP3A4.

In a pediatric study of 48 patients, higher dose of methylprednisolone was correlated with higher dosing requirement for tacrolimus to achieve concentrations within the therapeutic range, regardless of CYP $3 A 5$ genotype.${ }^{29}$ Higher dose of methylprednisolone was also associated with lower dose-normalized tacrolimus concentration independent of CYP3A5 genotype. This suggests that the effect of CYP $3 A 5$ genotype on drug interaction interindividual variability is drug specific. This may be related to the mechanism of drug interaction, in which methylprednisolone causes enzyme induction while fluconazole causes enzyme inhibition.

\section{Effect of CYP3A5 polymorphism on graft outcomes}

In one of the earliest studies reporting clinical outcomes, MacPhee et $\mathrm{al}^{27}$ found that in their cohort of 178 patients, those expressing CYP3A5 enzyme were significantly more likely to be below the target therapeutic range $(39.6 \%$ of patients) compared to CYP $3 A 5 * 3 / * 3$ genotypes $(8.8 \%$ of patients) in the first week posttransplant. Despite use of therapeutic drug monitoring, a significant segment of the CYP $3 A 5$ $* 1 / * 1$ or $* 1 / * 3$ populations ( $17 \%$ of patients) continued to have blood concentrations below the therapeutic range in the second week after transplant. Only $4 \%$ of patients with the $C Y P 3 A 5 * 3 / * 3$ genotype had subtherapeutic tacrolimus concentrations in the second week. The difference in achieving therapeutic drug concentrations did not translate into any observed differences in rejection rates. However, the authors did note that CYP3A5 expressers had earlier rejection compared to nonexpressers (median 7 versus 13 days, $p=0.005$ ). Tacrolimus target concentrations in this study were high (15-20 ng/ $\mu \mathrm{L}$ in the first week, $10-15 \mathrm{ng} / \mu \mathrm{L}$ in the second week) when compared to contemporary practice. Moreover, immunosuppression therapy consisted of dual therapy only without antibody induction. The overall rejection rate was high at $42.7 \%$. In the modern era, use of antibody induction is much more prevalent and may blunt the deleterious effect of subtherapeutic tacrolimus concentrations.

In a more recent study, CYP $3 A 5$ polymorphism had no impact on renal function as measured by estimated glomerular filtration rate, acute rejection rates, or tacrolimus toxicity (nephrotoxicity and neurotoxicity). ${ }^{62}$ This study involved only 103 renal transplant recipients. A larger study of 209 kidney transplant recipients similarly demonstrated no relationship between CYP $3 A 5$ genotype and renal function, biopsy-proven acute rejection rates, delayed graft function, or tacrolimus toxicity on biopsy ${ }^{44} \mathrm{~A}$ third study from Thailand enrolled 164 patients in the first 3 months posttransplant. The rate of biopsy-proven acute rejection and median time to first rejection episode was similar in both CYP3A5 expressers and nonexpressers. ${ }^{63}$ Recently, Flahault et al ${ }^{64}$ published the largest observational study of 577 patients followed for up to 5 years. There was no association of CYP $3 A 5$ genotype with biopsy result, renal function, biopsy-proven acute rejection, or graft survival. 
In a meta-analysis of 10 studies (1,246 patients), Terrazino et $\mathrm{al}^{28}$ found that there was no difference in acute rejection rate between $\mathrm{CYP} 3 \mathrm{~A} 5$ expressers and nonexpressers (OR: 0.763, 95\% CI: 0.532-1.094). On the other hand, in a newer and larger meta-analysis of 21 studies $(n=2,185)$, Rojas reported the pooled estimate showed a higher likelihood of acute rejection in patients who are CYP3A5 expressers (OR: 1.32, 95\% CI: 1.02-1.71) compared to nonexpressers. ${ }^{14}$ The majority of studies (15 of 21) used renal biopsy for diagnosis of acute rejection, while the remaining studies used clinical criteria or a combination of clinical criteria and biopsy. When limited to only studies with biopsy-proven acute rejection, the difference between CYP3A5 expressers versus nonexpressers no longer existed (OR: 1.25, 95\% CI: 0.96-1.62).

Overall, the evidence suggests little correlation between CYP3A5 genotype and clinically important outcomes. This observation may be explained by several reasons. First, deviations outside the therapeutic range due to genetic polymorphisms appear to play a role early posttransplant when the patient is first initiated on a weight-based tacrolimus dose. The use of therapeutic drug monitoring can easily correct sub- or supratherapeutic drug exposure quickly. Second, tacrolimus concentration is one of many contributing factors to acute rejection risk or graft function. Multiple other factors play a role. Existing studies may lack sufficient power to detect small differences in rejection risk related to CYP3A5 genotype solely. Third, in the early period posttransplant, concomitant immunosuppression in the form of antibody induction therapy, mycophenolate, and high dose corticosteroid is prevalent. Any negative impact of tacrolimus underdosing is mitigated by use of these other medications.

\section{Beyond CYP3A5: multivariable prediction of tacrolimus pharmacokinetics}

There is evidence that accounting for multiple genetic polymorphisms predicts tacrolimus clearance better than use of CYP $3 A 5$ genotype alone. In one study, Gervasini et $\mathrm{a}^{62}$ examined the relationship between both clinical outcomes and tacrolimus pharmacokinetics with $C Y P 3 A 4 * 1 B$, $C Y P 3 A 5 * 3, C Y P 2 C 8 * 3, C Y P 2 J 2 * 7$, and $A B C B 1$ genes. The authors found that $C Y P 3 A 4 * 1 B$ status additionally predicted tacrolimus pharmacokinetics on top of $C Y P 3 A 5$ genotype. A recent analysis used both $C Y P 3 A 4 * 22$ and $C Y P 3 A 5 * 3$ genotypes to categorize patients as poor, intermediate, or extensive metabolizers of tacrolimus. ${ }^{65}$ The combination of both genotypes was used to define optimal starting doses for each category of metabolizer. Another larger study looked at tacrolimus trough concentrations from 695 renal transplant recipients analyzed against a broad panel of more than 700 genetic variants. Although CYP $3 A 5$ was found to be the most important genetic variant, six additional variants were also found to each explain up to $2 \%-6 \%$ of the dosing variability in tacrolimus. ${ }^{12}$ The authors suggest that dosing algorithms based on multiple clinical and genetic factors work better than dosing based on CYP $3 A 5$ genotype alone. In a Han Chinese population, analysis of 10 SNPs covering 4 genes demonstrated that the CYP $3 A 5$ gene played the predominant role in determining dose-adjusted tacrolimus trough concentrations. ${ }^{66}$ However, in CYP3A5 nonexpressers only, additional SNPs in the genes coding for CYP3A4, MDR1, NR1I2 also predicted tacrolimus trough concentrations.

CYP3A5 genotype has been included as a parameter in pharmacokinetic modeling to predict tacrolimus dose requirement. In one externally validated model, the authors used the assumption that tacrolimus clearance was $30 \%$ higher (95\% CI: $13 \%-46 \%$ ) and bioavailability $18 \%$ lower (95\% CI: 2\%-29\%) in CYP3A5 expressers compared with nonexpressers. ${ }^{67} \mathrm{~A}$ dosing strategy based on Bayesian dose adjustment with this model successfully led to $65 \%$ of patients achieving mean tacrolimus concentrations within an acceptable target range, compared to $32 \%$ using a weight-based strategy. Not all dosing algorithms have been successful when applied to an external population. When the dosing algorithm published by Passey et a $1^{17,68}$ was implemented in an independent cohort, the algorithm was found to be poorly predictive of tacrolimus clearance. In this cohort of 255 patients, the correlation of dose-normalized whole blood trough concentrations had low correlation with clearance predicted by the equation from the algorithm.

Genetic variation in proteins associated with CYP activity may also impact tacrolimus disposition. Cytochrome P450 oxidoreductase (POR) is a protein that interacts with CYP through electron donation and is considered essential for CYP-mediated drug biotransformation. One SNP in particular, $P O R * 28($ rs 1057868; $\mathrm{C}>\mathrm{T})$, increases in vivo CYP3A activity. ${ }^{21}$ In one study of 71 healthy volunteers, $P O R * 28$ carriers who were CYP3A5 expressers had $40 \%$ lower tacrolimus exposure compared to CYP3A5 expressers with wild type $P O R{ }^{69}$ Interestingly, the population of CYP3A5 nonexpressers did not demonstrate any effect by POR SNP. In this case, drug metabolism is wholly dependent on CYP3A4, which does not interact in the same manner with POR. 


\section{Conclusion and future directions}

Over the past two decades, the role of CYP $3 A 5$ genetic polymorphism in influencing tacrolimus dose requirement has been extensively documented. The best studied polymorphism is the SNP 6986A $>$ G. The mutant variant bearing $G$ nucleotide is denoted by $* 3$ allele. In most ethnic populations, except blacks, homozygous *3/*3 is the most frequent genotype and is associated with CYP3A5 nonexpression. Tacrolimus clearance is approximately two-fold lower in nonexpressers, and dose-adjusted trough concentrations are higher. The wild-type variant bearing A nucleotide is denoted by * 1 allele and is associated with CYP3A5 enzyme expression. CYP3A5 expressing individuals have lower tacrolimus trough concentrations by $40 \%-50 \%$ when provided the standard weight-based dose. These individuals should be provided empiric doses $1.5-2$ times higher than the usual starting dose. Nevertheless, clinical use of genotype-based dosing for tacrolimus has not been widely adopted. To date, majority of studies have not demonstrated benefit in clinically important outcomes such as acute rejection, risk of nephrotoxicity, or graft survival with using genotype-based dosing. CYP $3 A 5$ genotype has been noted to impact patient response to drug interaction with fluconazole and differential absorption of drug formulations.

There is a lack of studies measuring clinical outcomes associated with use of multivariable dosing algorithms compared to weight-based dosing. Further studies are needed to clarify the effect of renal CYP $3 A 5$ genotype on risk of tacrolimus-induced nephrotoxicity. Currently available studies report conflicting results. Preliminary evidence suggests that epigenetic changes affect CYP3A4 and $3 \mathrm{~A} 5$ expression, but these have not been studied in human trials. ${ }^{70}$

\section{Disclosure}

The authors report no conflicts of interest in this work.

\section{References}

1. Staatz CE, Goodman LK, Tett SE. Effect of CYP3A and ABCB1 single nucleotide polymorphisms on the pharmacokinetics and pharmacodynamics of calcineurin inhibitors: part 2. Clin Pharmacokinet. 2010;49(4):207-221.

2. Webster A, Woodroffe RC, Taylor RS, Chapman JR, Craig JC. Tacrolimus versus ciclosporin as primary immunosuppression for kidney transplant recipients. Cochrane Database Syst Rev. 2005;(4):CD003961.

3. Hart A, Smith JM, Skeans MA, et al. OPTN/SRTR 2015 annual data report: kidney. Am J Transplant. 2017;17 (Supp 1):21-116.

4. Schiff J, Cole E, Cantarovich M. Therapeutic monitoring of calcineurin inhibitors for the nephrologist. Clin J Am Soc Nephrol. 2007;2(2):374-384.

5. Staatz CE, Tett SE. Clinical pharmacokinetics and pharmacodynamics of tacrolimus in solid organ transplantation. Clin Pharmacokinet. 2004;43(10):623-653.
6. Prograf® [package insert]. Northbrook, IL: Astellas Pharmaceuticals US, Inc.; 2015. Available from: https://www.astellas.us/docs/prograf. pdf. Accessed July 26, 2017.

7. Advagraf $®$ [product monograph]. Markham, ON: Astellas Pharma Canada, Inc.; 2010. Available from: http://www.astellas.ca/pdf/en/ monograph/2010-09-17AdvagrafProductMonograph-En.pdf. Accessed July 26, 2017.

8. Moller A, Iwasaki K, Kawamura A, et al. The disposition of 14C-labeled tacrolimus after intravenous and oral administration in healthy human subjects. Drug Metab Dispos. 1999;27:633-636.

9. Wilkinson GR. Drug metabolism and variability among patients in drug response. N Engl J Med. 2005;352:2211-2221.

10. Wrighton SA, Schuetz EG, Thummel KE, Shen DD, Korzekwa KR, Watkins PB. The human CYP3A subfamily: practical considerations. Drug Metab Rev. 2000;32(3-4):339-361.

11. Barry A, Levine M. A systematic review of the effect of CYP3A5 genotype on the apparent oral clearance of tacrolimus in renal transplant recipients. Ther Drug Monit. 2010;32(6):708-714.

12. Jacobson PA, Oetting WS, Brearley AM, et al. Novel polymorphisms associated with tacrolims trough concentrations: results from a multicenter kidney transplant consortium. Transplantation. 2011;91(3):300-308.

13. Birdwell KA, Decker B, Barbarino JM, et al. Clinical pharmacogenetics implementation consortium (CPIC) guidelines for CYP3A5 genotype and tacrolimus dosing. Clin Pharmacol Ther. 2015;98(1):19-24.

14. Rojas L, Neumann I, Herrero MJ, et al. Effect of CYP3A5*3 on kidney transplant recipients treated with tacrolimus: a systematic review and meta-analysis of observational studies. Pharmacogenomics J. 2015;15(1):38-48.

15. Tang JT, Andrew LM, van Gelder T, et al. Pharmacogenetics aspects of the use of tacrolimus in renal transplantation: recent developments and ethnic considerations. Expert Opin Drug Metab Toxicol. 2016;12(5):555-565.

16. Chen P, Li J, Li J, et al. Dynamic effects of CYP3A5 polymorphism on dose requirement and trough concentration of tacrolimus in renal transplant recipients. J Clin Pharm Ther. 2017;42(1):93-97.

17. Boughton O, Borgulya G, Cecconi M, Frederick S, Moreton-Clack M, MacPhee IA. A published pharmacogenetic algorithm was poorly predictive of tacrolimus clearance in an independent cohort of renal transplant recipients. Br J Clin Pharmacol. 2013;76(3):425-431.

18. Niioka T, Kagya H, Saito M, et al. Capability of utilizing CYP3A5 polymorphisms to predict therapeutic dosage of tacrolimus at early stage post-renal transplantation. Int J Mol Sci. 2015;16(1):1840-1854.

19. Chandel N, Aggarwal PK, Minz M, Sakhuja V, Kohli KK, Jha V. CYP3A $5 * 1 / * 3$ genotype influences the blood concentration of tacrolimus in response to metabolic inhibition by ketoconazole. Pharmacogenet Genomics. 2009;19(6):458-463.

20. Satoh S, Saito M, Inoue T, et al. CYP3A5 *1 allele associated with tacrolimus trough concentrations but not subclinical acute rejection or chronic allograft nephropathy in Japanese renal transplant recipients. Eur J Clin Pharmacol. 2009;65(5):473-481.

21. Hesselink DA, Bouamar R, Elens L, van Schaik RH, van Gelder T. The role of pharmacogenetics in the disposition of and response to tacrolimus in solid organ transplantation. Clin Pharmacokinet. 2014;53(2): 123-139.

22. Kuehl P, Zhang J, Lin Y, et al. Sequence diversity in CYP3A promoters and characterization of the genetic basis of polymorphic CYP3A5 expression. Nat Genet. 2001;27(4):383-391.

23. Staatz CE, Goodman LK, Tett SE. Effect of CYP3A and ABCB1 single nucleotide polymorphisms on the pharmacokinetics and pharmacodynamics of calcineurin inhibitors: part 1. Clin Pharmacokinet. 2010;49(3):141-175.

24. Hesselink DA, van Gelder T, van Schaik RH, et al. Population pharmacokinetics of cyclosporine in kidney and heart transplant recipients and the influence of ethnicity and genetic polymorphisms in the MDR-1, CYP3A4, and CYP3A5 genes. Clin Pharmacol Ther. 2004;76(6):545-556. 
25. Shi WL, Tang HL, Zhai SD. Effects of the CYP3A4*1B genetic polymorphism on the pharmacokinetics of tacrolimus in adult renal transplant recipients: a meta-analysis. PLoS One. 2015;10(6):e0127995.

26. Oetting WS, Schladt DP, Guan W, et al. Genomewide association study of tacrolimus concentrations in African American kidney transplant recipients identifies multiple CYP3A5 alleles. Am J Transplant. 2016;16(2):574-582.

27. MacPhee IA, Fredericks S, Tai T, et al. The influence of pharmacogenetics on the time to achieve target tacrolimus concentrations after kidney transplantation. Am J Transplant. 2004;4(6):914-919.

28. Terrazzino S, Quaglia M, Stratta P, Canonico PL, Genazzani AA. The effect of CYP3A5 6986A $>\mathrm{G}$ and $\mathrm{ABCB} 13435 \mathrm{C}>\mathrm{T}$ on tacrolimus dose-adjusted trough levels and acute rejection rates in renal transplant patients: a systematic review and meta-analysis. Pharmacogenet Genomics 2012;22(8):642-645.

29. Ferraris JR, Argibay PF, Costa L, et al. Influence of CYP3A5 polymorphism on tacrolimus maintenance doses and serum levels after renal transplantation: age dependency and pharmacological interaction with steroids. Pediatr Transplant. 2011;15(5):525-532.

30. Yong CJ, Jung LY, Bok JS, Ahyoung LL, Soo PM, Hwan KK. CYP3A5*3 genotype associated with intrasubject pharmacokinetic variation toward tacrolimus in bioequivalence study. Ther Drug Monit. 2010;32(1):67-72.

31. Spierings N, Holt DW, MacPhee IA. CYP3A5 genotype had no impact on intrapatient variability of tacrolimus clearance in renal transplant recipients. Ther Drug Monit. 2013;35(3):328-331.

32. Chu XM, Hao HP, Wang GJ, et al. Influence of CYP3A5 genetic polymorphism on cyclosporine A metabolism and elimination in Chinese renal transplant recipients. Acta Pharmacol Sin. 2006;27(11):1504-1508.

33. Hu YF, Qiu W, Liu ZQ, et al. Effects of genetic polymorphisms of CYP3A4, CYP3A5 and MDR1 on cyclosporine pharmacokinetics after renal transplantation. Clin Exp Pharmacol Physiol. 2006;33(11): 1093-1098.

34. Qiu XY, Jiao Z, Zhang M, et al. Association of MDR1, CYP3A4*18B, and CYP3A5*3 polymorphisms with cyclosporine pharmacokinetics in Chinese renal transplant recipients. Eur J Clin Pharmacol. 2008;64(11):1069-1084.

35. Anglicheau D, Thervet E, Etienne I, et al. CYP3A5 and MDR1 genetic polymorphisms and cyclosporine pharmacokinetics after renal transplantation. Clin Pharmacol Ther. 2004;75(5):422-433.

36. Hesselink DA, van Schaik RH, van der Heiden IP, et al. Genetic polymorphisms of the CYP3A4, CYP3A5, and MDR-1 genes and pharmacokinetics of the calcineurin inhibitors cyclosporine and tacrolimus. Clin Pharmacol Ther. 2003;74(3):245-254.

37. Zhao Y, Song M, Guan D, et al. Genetic polymorphisms of CYP3A5 genes and concentration of cyclosporine and tacrolimus. Transplant Proc. 2005;37(1):178-181.

38. Loh PT, Lou HX, Zhao Y, et al. Significant impact of gene polymorphisms on tacrolimus but not cyclosporine dosing in Asian renal transplant recipients. Transplant Proc. 2008;40(5):1690-1695.

39. Barbarino JM, Staatz CE, Venkataramanan R, et al. PharmGKB summary: cyclosporine and tacrolimus pathways. Pharmacogenet Genomics. 2013;23(10):563-585.

40. Wang P, Mao Y, Razo J, et al. Using genetic and clinical factors to predict tacrolimus dose in renal transplant recipients. Pharmacogenomics. 2010;11(10):1389-1402.

41. Thervet E, Loriot MA, Barbier S, et al. Optimization of initial tacrolimus dose using pharmacogenetic testing. Clin Pharmacol Ther. 2010;87(6):721-726.

42. Shuker N, Bouamar R, van Schaik RHN, et al. A randomized controlled trial comparing the efficacy of Сyp3a5 genotype-based with body weight-based tacrolimus dosing after living donor kidney transplantation. Am J Transplant. 2016;16(7):2085-2096.

43. Tacrolimus. Annotation of CPIC Guideline for tacrolimus and CYP3A5. Available from: https://www.pharmgkb.org/chemical/PA451578/guideline/PA166124619. Accessed February 15, 2018.
44. Glowacki F, Lionet A, Buob D, et al. CYP3A5 and ABCB1 polymorphisms in donor and recipient: impact on tacrolimus dose requirements and clinical outcome after renal transplantation. Nephrol Dial Transplant. 2011;26(9):3046-3050.

45. Givens RC, Lin YS, Dowling AL, et al. CYP3A5 genotype predicts renal CYP3A activity and blood pressure in healthy adults. J Appl Physiol. 2003;95(3):1297-1300.

46. Naesens M, Kuypers DR, Sarwal M. Calcineurin inhibitor nephrotoxicity. Clin J Am Soc Nephrol. 2009;4(2):481-508.

47. Quaglia M, Terrazzino S, Boldorini R, Stratta P, Genazzani AA. Severe acute nephrotoxicity in a kidney transplant patient despite low tacrolimus levels: a possible interaction between donor and recipient genetic polymorphisms. J Clin Pharm Ther. 2013;38(4):333-336.

48. Kuypers DR, Naesens M, de Jonge H, Lerut E, Verbeke K, Vanrenterghem Y. Tacrolimus dose requirements and CYP3A5 genotype and the development of calcineurin inhibitor-associated nephrotoxicity in renal allograft recipients. Ther Drug Monit. 2010;32(4):394-404.

49. Klauke B, Wirth A, Zittermann A, et al. No association between single nucleotide polymorphisms and the development of nephrotoxicity after orthotopic heart transplantation. $J$ Heart Lung Transplant. 2008;27(7):741-745.

50. Quteineh L, Verstuyft C, Furlan V, et al. Influence of CYP3A5 genetic polymorphism on tacrolimus daily dose requirements and acute rejection in renal graft recipients. Basic Clin Pharmacol Toxicol. 2008;103(6):546-552.

51. Fukudo M, Yano I, Yoshimura A, et al. Impact of MDR1 and CYP3A5 on the oral clearance of tacrolimus and tacrolimus-related renal dysfunction in adult living-donor liver transplant patients. Pharmacogenet Genomics. 2008;18(5):413-423.

52. Grenda R, Prokurat S, Ciechanowicz A, Piatosa B, Kalicinski P. Evaluation of the genetic background of standard-immunosuppressant-related toxicity in a cohort of 200 paediatric renal allograft recipients--a retrospective study. Ann Transplant. 2009;14(3):18-24.

53. Torio A, Aruyanet I, Montes-Ares O, et al. Effect of CYP 3 A $5 * 1 / * 3$ polymorphism on blood pressure in renal transplant recipients. Transplant Proc. 2012;44(9):2596-2598.

54. Ho ET, Wong G, Craig JC, Chapman JR. Once-daily extendedrelease versus twice-daily standard-release tacrolimus in kidney transplant recipients: a systematic review. Transplantation. 2013;95(9):1120-1128.

55. Fan B, Qiu K, Jiang Y, et al. Prograf produces more benefits for CYP3A5 low expression patients in early stage after kidney transplantation. Biomed Pharmacother. 2017;88:738-744.

56. Satoh S, Niioka T, Kagaya H, et al. Pharmacokinetic and CYP3A5 pharmacogenetic differences between once- and twice-daily tacrolimus from the first dosing day to 1 year after renal transplantation. Pharmacogenomics. 2014;15(11):1495-1506.

57. Zaltzman AS, Glick LA, Zaltzman JS, Nash M, Huang M, Prasad GV. The role of CYP3A5 polymorphism and dose adjustments following conversion of twice-daily to once-daily tacrolimus in renal transplant recipients. Transplant Res. 2016;5:2. eCollection 2016.

58. Lampen A, Christians U, Guengerich FP, et al. Metabolism of the immunosuppressant tacrolimus in the small intestine: cytochrome P450, drug interactions, and interindividual variability. Drug Metab Dispos. 1995;23(12):1315-1324.

59. Mori T, Kato J, Yamane A, et al. Drug interaction between voriconazole and tacrolimus and its association with the bioavailability of oral voriconazole in recipients of allogeneic hematopoietic stem cell transplantation. Int J Hematol. 2012;95(5):564-569.

60. Kuypers DR, de Jonge H, Naesens M, Vanrenterghem Y. Effects of CYP3A5 and MDR1 single nucleotide polymorphisms on drug interactions between tacrolimus and fluconazole in renal allograft recipients. Pharmacogenet Genomics. 2008;18(10):861-868.

61. Gibbs MA, Thummel KE, Shen DD, Kunze KL. Inhibition of cytochrome P-450 3A (CYP3A) in human intestinal and liver microsomes: comparison of $\mathrm{Ki}$ values and impact of CYP3A5 expression. Drug Metab Dispos. 1999;27(2):180-187. 
62. Gervasini G, Garcia M, Macias RM, Cubero JJ, Caravaca F, Benitez J. Impact of genetic polymorphisms on tacrolimus pharmacokinetics and the clinical outcome of renal transplantation. Transpl Int. 2012;25(4):471-480.

63. Yaowakulpatana K, Vadcharavivad S, Ingsathit A, et al. Impact of CYP3A5 polymorphism on trough concentrations and outcomes of tacrolimus minimization during the early period after kidney transplantation. Eur J Pharmacol. 2016;72(3):277-283.

64. Flahault A, Anglicheau D, Loriot MA, Thervet E, Pallet N. Clinical impact of the CYP3A5 6986A $>$ G allelic variant on kidney transplantation outcomes. Pharmacogenomics. 2017;18(2):165-173.

65. Woillard JB, Mourad M, Neely M, et al. Tacrolimus updated guidelines through popPK modeling: how to benefit more from CYP3A pre-emptive genotyping prior to kidney transplantation. Front Pharmacol. 2017;8:358.

66. Li JL, Liu S, Fu Q, et al. Interactive effects of CYP3A4, CYP3A5, MDR1 and NR1I2 polymorphisms on tracrolimus trough concentrations in early postrenal transplant recipients. Pharmacogenomics. 2015;16(12):1355-1365.
67. Storset E, Holford N, Hennig S, et al. Improved prediction of tacrolimus concentrations early after kidney transplantation using theory-based pharmacokinetic modelling. Br J Clin Pharmacol. 2014;78(3):509-523.

68. Passey C, Birnbaum AK, Brundage RC, Oetting WS, Israni AK, Jacobson PA. Dosing equation for tacrolimus using genetic variants and clinical factors. Br J Clin Pharmacol. 2011;72(6):948-957.

69. Zhang JJ, Zhang H, Ding XL, Ma S, Miao LY. Effect of the P450 oxidoreductase 28 polymorphism on the pharmacokinetics of tacrolimus in Chinese healthy male volunteers. Eur J Pharmacol. 2013;69(4): $807-812$.

70. Zhu H, Ge W. Future of the pharmacogenomics of calcineurin inhibitors in renal transplant patients. Pharmacogenomics. 2011;12(11): 1505-1508.
Pharmacogenomics and Personalized Medicine

\section{Publish your work in this journal}

Pharmacogenomics and Personalized Medicine is an international, peerreviewed, open access journal characterizing the influence of genotype on pharmacology leading to the development of personalized treatment programs and individualized drug selection for improved safety, efficacy and sustainability. This journal is indexed on the American Chemical

\section{Dovepress}

Society's Chemical Abstracts Service (CAS). The manuscript management system is completely online and includes a very quick and fair peer-review system, which is all easy to use. Visit http://www.dovepress. com/testimonials.php to read real quotes from published authors. 